\title{
MONOIDAL MORITA EQUIVALENCE
}

\author{
KORNÉL SZLACHÁNYI
}

Let $A$ be an algebra over the commutative ring $k$. It is well known that the category $\mathrm{M}_{A}$ of right $A$-modules is cocomplete, Abelian and the right regular object $A_{A}$ is a small projective generator. The latter three properties means precisely that the functor $\operatorname{Hom}_{A}\left(A,,_{-}\right): \mathrm{M}_{A} \rightarrow \mathrm{M}_{k}$ preserves coproducts, preserves cokernels and it is faithful, respectively. In fact this functor is monadic and has a right adjoint. It is also well known 8 that the above properties characterize such categories: For a $k$-linear category $\mathcal{C}$ to be equivalent to $\mathrm{M}_{A}$ for some $k$-algebra $A$ it is sufficient that $\mathcal{C}$ is cocomplete, Abelian and possesses a small projective generator. Of course the algebra $A$ is determined by $\mathcal{C}$ only up to Morita equivalence. The analogue question for monoidal module categories has been studied by B. Pareigis in [10. With the advent of quantum groupoids it is worth reconsidering the question.

Therefore we are interested in monoidal structures on $M_{A}$ admitting a strong monoidal forgetful functor to the category ${ }_{R} \mathrm{M}_{R}$ of bimodules over some other $k$ algebra $R$. In the special case of $R=k$ one obtains that $A$ is a bialgebra [11. The general case leads to bialgebroids [12. The importance of module categories of bialgebroids, as well as those of Hopf algebroids, weak bialgebras and weak Hopf algebras, is that they provide examples of non-Tannakian monoidal categories.

In a recent paper [16] the forgetful functors of bialgebroids over $R$ have been characterized abstractly as the strong monoidal monadic functors to ${ }_{R} \mathrm{M}_{R}$ with a right adjoint. The bicategory of bialgebroids proposed in [16 is based on a comparison of certain monads on the base categories ${ }_{R} \mathrm{M}_{R}$. Unfortunately, it does not admit a comparison of the monoidal categories $\mathrm{M}_{A}$ directly, without knowing at least a functor between their base categories. In Section 2 a new construction of a bicategory of bialgebroids will be given which is completely base independent and in which the equivalence of two objects can be interpreted as monoidal Morita equivalence. The basic idea can be found already in Takeuchi's paper 18. This is why our monoidal Morita equivalence reduces to $\sqrt{\text { Morita }}$ equivalence in case of the Sweedler bialgebroids $E(R)$ which are the bialgebroid structures on the Sweedler corings $R^{e}=R^{\mathrm{op}} \otimes R$. Note that the monoidal version of classical Morita theory, what we study here, is a priori different from (weak or not weak) Morita theory for monoidal categories 9 .

The results of Section [5 put forward the notion of strong comonoid progenerators: They classify the members of a monoidal Morita equivalence class and their existence characterize the categories of modules over a bialgebroid. Similarly, existence of strong Frobenius progenerators characterize the categories $\mathrm{M}_{A}$ with $A$ being a Frobenius Hopf algebroid.

\section{The BiCATEgory of COALGEBroids}

We fix a commutative ring $k$ throughout the paper. All categories and functors are assumed to be $k$-linear even if not stated explicitly. 
In his paper [18] M. Takeuchi introduced the notion of an $R \mid S$-coring that play the role of 1-cells in a bicategory which we are going to describe.

Definition 1.1. For $k$-algebras $R$ and $S$ an $R \mid S$-coalgebroid is an $R \otimes S$ - $R \otimes$ $S$-bimodule $C$ with an $S$-coring structure $\langle C, \Delta, \varepsilon\rangle$ such that $\Delta$ is also an $R$ - $R$ bimodule map and its image is in the $R$-centralizer $(C \underset{S}{\otimes} C)^{R}$. That is to say, using the familiar notation $\Delta(c)=c^{(1)} \otimes c^{(2)}$,

$$
\begin{aligned}
\Delta\left(r . c \cdot r^{\prime}\right) & =c^{(1)} \cdot r^{\prime} \otimes_{S} r \cdot c^{(2)} \\
r . c^{(1)} \otimes_{S} c^{(2)} & =c^{(1)} \otimes_{S} c^{(2)} \cdot r
\end{aligned}
$$

for all $c \in C$ and $r, r^{\prime} \in R$.

We denote the $S$-actions by upper dots and the $R$-actions by lower dots which becomes important in the special case when $R=S$. In this case the ambiguous $(C \otimes C)^{S}$ is denoted by $C \times C$. It follows from (1.2) that

$$
\varepsilon(r . c)=\varepsilon(c . r) \quad r \in R, c \in C .
$$

Definition 1.2. Let $\left\langle P, \Delta_{P}, \varepsilon_{P}\right\rangle$ and $\left\langle Q, \Delta_{Q}, \varepsilon_{Q}\right\rangle$ be $R \mid S$-coalgebroids. A $k$-linear map $\alpha: P \rightarrow Q$ satisfying

$$
\begin{aligned}
\alpha\left(s \cdot p \cdot s^{\prime}\right) & =s \cdot \alpha(p) \cdot s^{\prime} \\
\alpha\left(r \cdot p \cdot r^{\prime}\right) & =r \cdot \alpha(p) \cdot r^{\prime} \\
\left(\alpha \otimes_{S} \alpha\right) \circ \Delta_{P} & =\Delta_{Q} \circ \alpha \\
\varepsilon_{Q} \circ \alpha & =\varepsilon_{P}
\end{aligned}
$$

is called a map of coalgebroids. The $R \mid S$-coalgebroids and $R \mid S$-coalgebroid maps form a category $\operatorname{Cgd}(S, R)$.

Definition 1.3. Let Cgd be the bicategory with

(1) objects being the $k$-algebras $R, S, \ldots$,

(2) hom-categories $\operatorname{Cgd}(S, R)$,

(3) horizontal composition $\operatorname{Cgd}(S, R) \times \operatorname{Cgd}(T, S) \rightarrow \operatorname{Cgd}(T, R)$ being given

- for objects by $\langle P, Q\rangle \mapsto P \odot Q$ where the $R \mid T$-coalgebroid $P \odot Q$ has underlying multimodule

$$
\begin{aligned}
P \odot Q & =P \otimes_{S^{e}} Q \\
& \equiv P \otimes Q /\left\{s \cdot p \cdot s^{\prime} \otimes q-p \otimes s^{\prime} \cdot q \cdot s\right\} \\
t \cdot(p \odot q) \cdot t^{\prime} & =p \odot\left(t \cdot q \cdot t^{\prime}\right) \\
r \cdot(p \odot q) \cdot r^{\prime} & =\left(r \cdot p \cdot r^{\prime}\right) \odot q
\end{aligned}
$$

and comultiplication and counit

$$
\begin{aligned}
\Delta_{P \odot Q}(p \odot q) & =p^{(1)} \odot q^{(1)} \otimes_{T} p^{(2)} \odot q^{(2)} \\
\varepsilon_{P \odot Q}(p \odot q) & =\varepsilon_{Q}\left(\varepsilon_{P}(p) \cdot q\right)
\end{aligned}
$$

- and for arrows $\alpha: P \rightarrow P^{\prime}: S \rightarrow R$ and $\beta: Q \rightarrow Q^{\prime}: T \rightarrow S$ by

$$
(\alpha \odot \beta)(p \odot q)=\alpha(p) \odot \beta(q)
$$


(4) and with horizontal unit $E(R)$ at the object $R$ being the Sweedler coring $R^{e}$ as the $R \mid R$-coring with underlying multimodule

$$
\begin{aligned}
E(R) & =R \otimes R \\
r \cdot\left(r_{1} \otimes r_{2}\right) \cdot r^{\prime} & =r r_{1} \otimes r_{2} r^{\prime} \\
r \cdot\left(r_{1} \otimes r_{2}\right) \cdot r^{\prime} & =r_{1} r^{\prime} \otimes r r_{2}
\end{aligned}
$$

and coring structure

$$
\begin{aligned}
\Delta_{E(R)}\left(r_{1} \otimes r_{2}\right) & =\left(r_{1} \otimes 1_{R}\right){\underset{R}{(}}_{R}\left(1_{R} \otimes r_{2}\right) \\
\varepsilon_{E(R)}\left(r_{1} \otimes r_{2}\right) & =r_{1} r_{2} .
\end{aligned}
$$

The coherence isomorphisms

$$
O \odot(P \odot Q) \stackrel{\sim}{\longrightarrow}(O \odot P) \odot Q \quad \text { for } U \stackrel{Q}{\longrightarrow} T \stackrel{P}{\longrightarrow} S \stackrel{O}{\longrightarrow} R
$$

and

$$
E(R) \odot P \stackrel{\sim}{\longrightarrow} P, \quad P \odot E(S) \stackrel{\sim}{\longrightarrow} P \quad \text { for } S \stackrel{P}{\longrightarrow} R
$$

are not written out explicitly. It suffices to note that they are the "same" as the coherence isomorphisms in the bicategory $\mathbb{B} \mathbb{M}\left(\mathrm{M}_{k}\right)$ of bimodules after forgetting the coring structure and considering the 1-cells $P: S \rightarrow R$ merely as $R^{e}-S^{e}$-bimodules. This can be formulated by saying that the forgetful functor

$$
\mathrm{U}: \mathrm{Cgd} \rightarrow \mathbb{B I M}\left(\mathrm{M}_{k}\right)
$$

sending $\left\langle R_{R^{e}} P_{S^{e}}, \Delta_{P}, \varepsilon_{P}\right\rangle$ to $R_{R^{e}} P_{S^{e}}$ is a strict homomorphism of bicategories.

\section{The Bicategory Bgd}

Bialgebroids have several, sligtly different though equivalent, formulations in the literature [17, 7, 15, 2, 3,. For our purposes the following definition is the most appropriate: A bialgebroid over $R$ is a monoid $\langle A, \mu, \eta\rangle$ in the monoidal category $\operatorname{Cgd}(R, R)$. Especially, the monoidal unit $E(R)$ of $\operatorname{Cgd}(R, R)$ considered with the coherence isomorphism $E(R) \odot E(R) \stackrel{\sim}{\longrightarrow} E(R)$ as multiplication, i.e., with

$$
\left(r_{1} \otimes r_{2}\right) \otimes_{R^{e}}\left(r_{1}^{\prime} \otimes r_{2}^{\prime}\right) \mapsto\left(r_{1}^{\prime} r_{1} \otimes r_{2} r_{2}^{\prime}\right)
$$

is a bialgebroid in this sense. So a natural way to introduce 1-cells $P: A \rightarrow B$ between bialgebroids and 2-cells $\alpha: P \rightarrow Q: A \rightarrow B$ between such 1-cells is provided by considering the bicategory of bimodules in Cgd.

Definition 2.1. The bicategory Bgd of bialgebroids is defined as $\mathbb{B} \mathbb{I M}(\mathrm{Cgd})$. This means that the objects of Bgd are the monoids ${ }^{1}$ in Cgd, the 1-cells $P: A \rightarrow B$ are the $B$-A-bimodules in Cgd and the 2-cells $\alpha: P \rightarrow Q: A \rightarrow B$ are the $B$ - $A$ bimodule morphisms in Cgd. Horizontal composition of $B \stackrel{P}{\longrightarrow} A$ and $C \stackrel{Q}{\longrightarrow} B$ is denoted by $P \otimes Q$.

In the above definition we described the cells of Bgd in the language of Cgd. The more elementary language is that of $\mathbb{B I M}\left(\mathrm{M}_{k}\right)$ which we now use to describe $\mathrm{Bgd}$.

\footnotetext{
${ }^{1}$ We mean monads, i.e., endo-1-cells with monoid structure, and not pseudomonoids which would be structures on 0-cells.
} 
2.1. Bialgebroids. A bialgebroid $A$ over $R$ is a monoid $\langle A, \mu, \eta\rangle$ in $\operatorname{Cgd}(R, R)$. Therefore $A$ is a multimodule over $R$ with four actions denoted $r . a, r \cdot a, a . r$ and $a \cdot r$, respectively, and has a coring structure

$$
\begin{aligned}
\Delta_{A}: A & \rightarrow A \underset{R}{\otimes} A \\
\varepsilon_{A}: A & \rightarrow R
\end{aligned}
$$

where $\otimes_{R}$ is taken w.r.t. the upper dot actions. In order for $\langle: A:, \Delta, \varepsilon\rangle$ to be a coalgebroid the structure maps should satisfy

$$
\begin{aligned}
& \Delta_{A}\left(r \cdot a \cdot r^{\prime}\right)=a^{(1)} \cdot r^{\prime}{\underset{R}{(2)} r}_{a^{(2)}} \\
& r . a^{(1)} \otimes_{R} a^{(2)}=a^{(1)}{\underset{R}{\otimes}}^{(2)} \cdot r
\end{aligned}
$$

for all $a \in A$ and $r, r^{\prime} \in R$.

The forgetting functor $\mathbf{U}$ applied to the monoid $\langle A, \mu, \eta\rangle$ gives an $R^{e}$-ring. This means that $A$ is a $k$-algebra with multiplication $\left\langle a, a^{\prime}\right\rangle \mapsto a a^{\prime}$ and unit element $1_{A}$ and the $\eta$ is a $k$-algebra map $R^{e} \rightarrow A$. So we can write

$$
\eta=t_{A} \otimes s_{A}: R^{\mathrm{op}} \otimes R \rightarrow A
$$

with uniquely determined algebra maps $s_{A}: R \rightarrow A$ and $t_{A}: R^{\mathrm{op}} \rightarrow A$ of commuting ranges.

But $\eta$ is also a 2-cell $E(R) \rightarrow A$ in Cgd therefore it is a multimodule map

$$
r \cdot\left(1_{A}\right) \cdot r^{\prime}=t_{A}(r) s_{A}\left(r^{\prime}\right)=r^{\prime} \cdot\left(1_{A}\right) \cdot r \quad r, r^{\prime} \in R
$$

and a coring map. Knowing already that $\Delta_{A}$ and $\varepsilon_{A}$ belong to ${ }_{R} \mathrm{M}_{R}$, the latter reduces to the unitality conditions

$$
\Delta_{A}\left(1_{A}\right)=1_{A} \underset{R}{\otimes} 1_{A}, \quad \varepsilon_{A}\left(1_{A}\right)=1_{R} .
$$

The $\mu$ being a 2-cell $A \odot A \rightarrow A$ it is a multimodule map

$$
\begin{aligned}
& \left(r \cdot a \cdot r^{\prime}\right) a^{\prime}=r \cdot\left(a a^{\prime}\right) \cdot r^{\prime} \\
& a\left(r \cdot a^{\prime} \cdot r^{\prime}\right)=r \cdot\left(a a^{\prime}\right) \cdot r^{\prime}
\end{aligned}
$$

and it is compatible with the coring structures therefore

$$
\begin{aligned}
a^{(1)} a^{\prime(1)}{\underset{R}{\otimes}}^{(2)} a^{\prime(2)} & =\left(a a^{\prime}\right)^{(1)} \underset{R}{\otimes}\left(a a^{\prime}\right)^{(2)} \\
\varepsilon_{A}\left(a a^{\prime}\right) & =\varepsilon_{A}\left(\varepsilon_{A}(a) \cdot a^{\prime}\right) .
\end{aligned}
$$

Combining (2.3) and (2.4) with (2.1) then imply

$$
\begin{aligned}
& r \cdot a \cdot r^{\prime}=a t_{A}(r) s_{A}\left(r^{\prime}\right) \\
& r \cdot a \cdot r^{\prime}=s_{A}(r) t_{A}\left(r^{\prime}\right) a
\end{aligned}
$$

Summarizing: A monoid $\langle A, \mu, \eta\rangle$ in $\operatorname{Cgd}(R, R)$ is the same thing as a (right) bialgebroid $\left\langle A, R, s_{A}, t_{A}, \Delta_{A}, \varepsilon_{A}\right\rangle$ satisfying the axioms given e.g. in [15]. 
2.2. Bialgebroid morphisms. A 1-cell $P: B \rightarrow A$ from the bialgebroid $B$ over $S$ to the bialgebroid $A$ over $R$ has been defined as an $A$ - $B$-bimodule in $\operatorname{Cgd}(S, R)$, i.e., a triple

$$
\begin{gathered}
P \in \operatorname{Cgd}(S, R) \\
\lambda_{P}: A \odot P \rightarrow P \\
\rho_{P}: P \odot B \rightarrow P
\end{gathered}
$$

satisfying five commutative diagrams, as usual for bimodules. Forgetting via $\mathbf{U}$ this bimodule becomes an ordinary bimodule ${ }_{A} P_{B}$ in the (one object) bicategory $\mathrm{M}_{k}$. So $P$ is a $k$-module with left and right actions $\langle a, p\rangle \mapsto a \triangleright p$ and $\langle p, b\rangle \mapsto p \triangleleft b$ of the $k$-algebras $A$ and $B$, respectively. The multimodule structures on $A$ and $B$ allow to recognize the multimodule structure on $P$ because

$$
\begin{aligned}
& r \cdot p \cdot r^{\prime}=s_{A}(r) t_{A}\left(r^{\prime}\right) \triangleright p \\
& s \cdot p \cdot s^{\prime}=p \triangleleft t_{B}(s) s_{B}\left(s^{\prime}\right) .
\end{aligned}
$$

The forgotten structures amount to have $S$ - $S$ bimodule maps

$$
\begin{gathered}
\Delta_{P}: P \rightarrow P \underset{S}{\otimes} P \\
\varepsilon_{P}: P \rightarrow S
\end{gathered}
$$

satisfying comonoid axioms in ${ }_{S} \mathrm{M}_{S}$ and

$$
\begin{aligned}
& r \cdot p^{(1)} \underset{S}{\otimes} p^{(2)}=p^{(1)} \underset{S}{\otimes} p^{(2)} \cdot r \\
& \Delta_{P}(a \triangleright p \triangleleft b)=a^{(1)} \triangleright p^{(1)} \triangleleft b^{(1)}{\underset{S}{S} a^{(2)} \triangleright p^{(2)} \triangleleft b^{(2)}}(a \triangleright p \triangleleft b)=\varepsilon_{B}\left(\varepsilon_{P}\left(\varepsilon_{A}(a) \cdot p\right) \cdot b\right)
\end{aligned}
$$

Note that the last two equations express the fact that the $\lambda_{P}$ and $\rho_{P}$ are 2-cells in Cgd. Bgd.

Regrouping the axioms one obtains the following description of the 1-cells of

Lemma 2.2. The triple $\left\langle P, \Delta_{P}, \varepsilon_{P}\right\rangle$ is a morphism of bialgebroids from $B$ over $S$ to $A$ over $R$ iff

(1) $P$ is an $A-B$ bimodule ${ }_{A} P_{B}$,

(2) $\left\langle P_{B}, \Delta_{P}, \varepsilon_{P}\right\rangle$ is a comonoid in $\left\langle\mathrm{M}_{B}, \otimes_{S}, S_{B}\right\rangle$,

(3) $\Delta_{P}: P \rightarrow(P \otimes P)^{R}$ and

(4) $\Delta_{P}(a \triangleright p)=a^{(1)} \triangleright p^{(1)}{\underset{S}{(2)}}^{(2)} \triangleright p^{(2)}$,

(5) $\varepsilon_{P}(a \triangleright p)=\varepsilon_{P}\left(\varepsilon_{A}(a) \cdot p\right)$

hold for all $a \in A$ and $p \in P$.

Loosely speaking, 1-morphisms $B \rightarrow A$ of bialgebroids are right $B$-module coalgebras with compatible left $A$-module structure.

2.3. Bialgebroid transformations. If both $P$ and $Q$ are bialgebroid morphisms from $B$ over $S$ to $A$ over $R$ then a 2-cell $\alpha: P \rightarrow Q: B \rightarrow A$ is nothing but an $A$ - $B$-bimodule map

$$
\alpha:{ }_{A} P_{B} \rightarrow{ }_{A} Q_{B}
$$


satisfying

$$
\begin{aligned}
\alpha\left(p^{(1)}\right) \underset{S}{\otimes} \alpha\left(p^{(2)}\right) & =\alpha(p)^{(1)}{\underset{S}{S}}_{\varepsilon^{\prime}} \alpha(p)^{(2)} \\
\varepsilon_{Q}(\alpha(p)) & =\varepsilon_{P}(p) .
\end{aligned}
$$

\section{Embedding into MonCat}

If $A$ is a right bialgebroid over $R$ then the category $\mathrm{M}_{A}$ of right $A$-modules has a unique monoidal structure $\left\langle\mathrm{M}_{A}, \otimes_{R}, R_{A}\right\rangle$ such that the forgetful functor $U_{A}: \mathrm{M}_{A} \rightarrow$ ${ }_{R} \mathrm{M}_{R}$ associated to the algebra $\stackrel{R}{m}$ ap $\eta: R^{e} \rightarrow A$ is strict monoidal. This is the object map of a morphism of bicategories.

Definition 3.1. Let $\mathcal{H}: \mathrm{Bgd} \rightarrow \mathrm{MonCat}^{\mathrm{op}}$ be the morphism of bicategories into the 2-category of monoidal categories, monoidal functors and monoidal natural transformations

(1) which maps the bialgebroid $A$ to the monoidal category $\mathrm{M}_{A}$,

(2) the 1-cell $P: B \rightarrow A$ to the monoidal functor

$$
\begin{aligned}
\mathcal{H}(P) & :=\operatorname{Hom}_{B}(P,-): \mathrm{M}_{B} \rightarrow \mathrm{M}_{A} \\
\mathcal{H}(P)_{M, N} & : \operatorname{Hom}_{B}(P, M) \underset{R}{\otimes} \operatorname{Hom}_{B}(P, N) \rightarrow \operatorname{Hom}_{B}(P, M{\underset{S}{S}} N) \\
\mu & \underset{R}{\otimes} \mapsto(\mu \underset{S}{\otimes} \nu) \circ \Delta_{P} \\
\mathcal{H}(P)_{0} & : R_{A} \rightarrow \operatorname{Hom}_{B}(P, S) \\
r & \mapsto \varepsilon_{P}\left(r . ._{-}\right)
\end{aligned}
$$

(3) and the 2-cell $\alpha: P \rightarrow Q: B \rightarrow A$ to the monoidal natural transformation

$$
\operatorname{Hom}_{B}(\alpha, M): \operatorname{Hom}_{B}(Q, M) \rightarrow \operatorname{Hom}_{B}(P, M) .
$$

For each $C \stackrel{Q}{\longrightarrow} B \stackrel{P}{\longrightarrow} A$ the natural isomorphism

$$
\operatorname{Hom}_{B}\left(P, \operatorname{Hom}_{C}(Q, M)\right) \stackrel{\sim}{\longrightarrow} \operatorname{Hom}_{C}(P \underset{B}{\otimes} Q, M)
$$

and for each $A$ the natural isomorphism

$$
\mathrm{M}_{A} \stackrel{\sim}{\longrightarrow} \operatorname{Hom}_{A}\left(A,_{-}\right)
$$

endow $\mathcal{H}$ with the structure of a homomorphism of bicategories $[6]$.

Lemma 3.2. $\mathcal{H}$ is locally faithful and full.

Proof. Let $\alpha: P \rightarrow Q: B \rightarrow A$ be a 2-cell and assume that $\mathcal{H}(\alpha)=0$. Then $\mu \circ \alpha=0$ for all $\mu \in \operatorname{Hom}_{B}(Q, M)$ and for all $M \in \mathrm{M}_{B}$. Choosing $M=Q$ and $\mu=Q$ we obtain $\alpha=0$. Now let $\kappa: \mathcal{H}(Q) \rightarrow \mathcal{H}(P)$ be any monoidal natural transformation. Then Yoneda Lemma implies that there is an $A$ - $B$-bimodule map $\alpha: P \rightarrow Q$ such that $\kappa_{M}=\operatorname{Hom}_{B}(\alpha, M)$. Since $\kappa$ is monoidal,

$$
\begin{aligned}
(\alpha \underset{S}{\otimes} \alpha) \circ \Delta_{P} & =\mathcal{H}(P)_{Q, Q} \circ\left(\kappa_{Q} \underset{R}{\otimes} \kappa_{Q}\right)(Q \underset{R}{\otimes} Q) \\
& =\kappa_{Q} \underset{S}{\otimes} \circ \mathcal{H}(Q)_{Q, Q}(Q \underset{R}{\otimes} Q)=\Delta_{Q} \circ \alpha
\end{aligned}
$$

and

$$
\varepsilon_{Q} \circ \alpha=\kappa_{S} \circ \mathcal{H}(Q)_{0}\left(1_{R}\right)=\mathcal{H}(P)_{0}\left(1_{R}\right)=\varepsilon_{P} .
$$

Thus $\alpha$ is a map of coalgebroids. So we have proven that the functor

$$
\mathcal{H}(B, A): \operatorname{Bgd}(B, A) \rightarrow \operatorname{MonCat}^{\mathrm{op}}\left(\mathrm{M}_{B}, \mathrm{M}_{A}\right)
$$


is full and faithful for any pair of bialgebroids $A, B$.

Lemma 3.3. Let $A$ and $B$ be bialgebroids over $R$ and $S$, respectively, and let $G: \mathrm{M}_{B} \rightarrow \mathrm{M}_{A}$ be a monoidal functor. Then $G$ is isomorphic to $\mathcal{H}(P)$ for some bialgebroid morphism $P: B \rightarrow A$ if and only if the underlying ordinary functor of $G$ has a left adjoint.

Proof. Clearly the functor $\operatorname{Hom}_{B}\left(P,_{-}\right)$has left adjoint, namely - $\otimes_{A} P$. Now assume that $\left\langle G, G_{2}, G_{0}\right\rangle$ is a monoidal functor and $F \dashv G$ is a left adjoint. Then $F$ has an opmonoidal structure $\left\langle F, F^{2}, F^{0}\right\rangle$ and there is a monoidal natural isomorphism

$$
G(M) \cong \operatorname{Hom}_{A}(A, G(M)) \cong \operatorname{Hom}_{B}(F(A), M) \equiv \mathcal{H}(P)(M)
$$

where $P:=F(A)$ is an $A$ - $B$-bimodule and has coring structure

$$
\begin{aligned}
\Delta_{P} & =F^{A, A} \circ F\left(\Delta_{A}\right) \\
\varepsilon_{P} & =F^{0} \circ F\left(\varepsilon_{A}\right)
\end{aligned}
$$

which satisfies the compatibility conditions (2.11), (2.12) and(2.13).

The characterization of the objects $\mathcal{H}(A)$ within MonCat is postponed until Section 5

\section{Special Morphisms}

4.1. Bialgebroid maps. Let $A$ and $B$ be bialgebroids over $R$ and $S$, respectively. A bialgebroid map $\left\langle f, f_{0}\right\rangle: A \rightarrow B$ is a pair of algebra maps $f: A \rightarrow B$ and $f_{0}: R \rightarrow S$ such that

$$
\begin{aligned}
f \circ s_{A} & =s_{B} \circ f_{0} \\
f \circ t_{A} & =t_{B} \circ f_{0} \\
\left(f \otimes_{S} f\right) \circ \Delta_{A} & =\Delta_{B} \circ f \\
\varepsilon_{B} \circ f & =f_{0} \circ \varepsilon_{A}
\end{aligned}
$$

where notice that $f_{0}$ is uniquely determined by $f$ via $f_{0}=\varepsilon_{B} \circ f \circ s_{A}$. Therefore we shall often say that " $f$ is a bialgebroid map" without mentioning $f_{0}$.

The bialgebroids and bialgebroid maps form a category BgdMap and the notion of isomorphism in this category leads to the

Definition 4.1. Two bialgebroids $A$ over $R$ and $B$ over $S$ are called isomorphic if there exist bialgebroid maps $f: A \rightarrow B$ and $g: B \rightarrow A$ such that $f \circ g$ and $g \circ f$ are identities.

To any bialgebroid map $\left\langle f, f_{0}\right\rangle: A \rightarrow B$ we can associate a bialgebroid morphism $f^{*}$ as follows. As an $A$-B-bimodule it is $B$ with left action of $A$ induced by $f$. The coring structure is inherited from $B$. It is easy to check that the triple $\left\langle f^{*}=\right.$ $\left.{ }_{f(A)} B_{B}, \Delta_{f^{*}}=\Delta_{B}, \varepsilon_{f^{*}}=\varepsilon_{B}\right\rangle$ satisfy the axioms for a 1-cell in Bgd. As a matter 
of fact,

$$
\begin{aligned}
& r . b^{(1)}{\underset{S}{\otimes}}_{b^{(2)}}=f\left(s_{A}(r)\right) b^{(1)}{\underset{S}{\otimes}}_{b^{(2)}}=s_{B}\left(f_{0}(r)\right) b^{(1)}{\underset{S}{\otimes}}_{b^{(2)}} \\
& =b^{(1)} \otimes_{S} t_{B}\left(f_{0}(r)\right) b^{(2)}=b^{(1)} \otimes_{S} f\left(t_{A}(r)\right) b^{(2)} \\
& =b^{(1)} \otimes_{S} b^{(2)} \cdot r \\
& \Delta_{f^{*}}(a \triangleright b)=\Delta_{B}(f(a)) \Delta_{B}(b)=(f \underset{S}{\otimes} f)\left(\Delta_{A}(a)\right) \Delta_{B}(b) \\
& =a^{(1)} \triangleright b^{(1)} \otimes_{S} a^{(2)} \triangleright b^{(2)} \\
& \varepsilon_{f^{*}}(a \triangleright b)=\varepsilon_{B}(f(a) b)=\varepsilon_{B}\left(\varepsilon_{B}(f(a)) . b\right)=\varepsilon_{B}\left(f_{0}\left(\varepsilon_{A}(a)\right) . b\right) \\
& =\varepsilon_{f^{*}}\left(\varepsilon_{A}(a) \cdot b\right)
\end{aligned}
$$

4.2. The forgetful functors. For $A$ a bialgebroid over $R$ the algebra map $\eta$ : $R^{e} \rightarrow A$ defines a forgetful functor $U_{A}: \mathrm{M}_{A} \rightarrow \mathrm{M}_{R^{e}}$ which, when $\mathrm{M}_{R^{e}}$ is identified with the monoidal category ${ }_{R} \mathrm{M}_{R}$, is a strict monoidal functor. One expects that this functor is the same as $\mathcal{H}\left(\eta^{*}\right)$ for the 1-cell $\eta^{*}: A \rightarrow E(R)$ associated to the bialgebroid map $\eta: E(R) \rightarrow A$.

Proposition 4.2. Let $\langle A, \mu, \eta\rangle$ be a bialgebroid over $R$. Then

(1) $\eta: E(R) \rightarrow A$ is a bialgebroid map

(2) and there is a monoidal isomorphism $U_{A} \cong \mathcal{H}\left(\eta^{*}\right)$.

Proof. (1) $\eta$ is an algebra map $R^{e} \rightarrow A,\left(r_{1} \otimes r_{2}\right) \mapsto t_{A}\left(r_{1}\right) s_{A}\left(r_{2}\right)$. Writing simply $E$ for $E(R)$ we have $s_{E}(r)=1_{R} \otimes r$ and $t_{E}=r \otimes 1_{R}$ therefore $\eta_{0}:=\varepsilon_{A} \circ \eta \circ s_{E}: R \rightarrow R$ is the identity and we have

$$
\begin{aligned}
\eta \circ s_{E} & =s_{A} \\
\eta \circ t_{E} & =t_{A} \\
\left(\eta \otimes_{R} \eta\right) \circ \Delta_{E} & =\Delta_{A} \\
\varepsilon_{A} \circ \eta & =\varepsilon_{E}
\end{aligned}
$$

(2) The 1-morphism $\eta^{*}: A \rightarrow E(R)$ has underlying bimodule $R^{e} A_{A}$ with left action $\left(r_{1} \otimes r_{2}\right) \triangleright a=r_{2} \cdot a . r_{1}$. The functor $\mathcal{H}\left(\eta^{*}\right)$ maps the right $A$-module $M$ to $\operatorname{Hom}_{A}(A, M)$ therefore the right $R^{e}$-module maps

$$
\begin{gathered}
\nu_{M}: U_{A}(M) \rightarrow \mathcal{H}\left(\eta^{*}\right)(M) \\
m \mapsto\{a \mapsto m \triangleleft a\}
\end{gathered}
$$

which are natural in $M$ define the required natural isomorphism. It remains to show that $\nu$ is monoidal, i.e.,

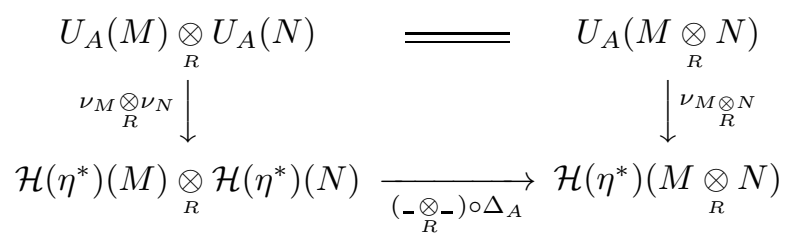


and

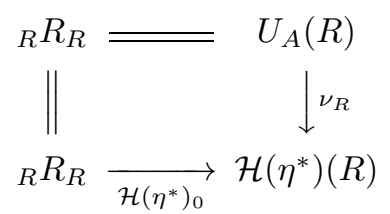

are commutative in ${ }_{R} \mathrm{M}_{R}$. The first diagram evaluated on $m \underset{R}{\otimes} n$ is equivalent to $(m \underset{R}{\otimes} n) \triangleleft a=\left(m \triangleleft a^{(1)}\right) \underset{R}{\otimes}\left(n \triangleleft a^{(2)}\right)$ and the second one is equivalent to the formula $r \triangleleft a=\varepsilon_{A}(r . a)$ for the trivial representation.

4.3. When $\mathcal{H}(P)$ is strong monoidal. Let $P: B \rightarrow A$ be a morphism of bialgebroids and assume that $\mathcal{H}(P): \mathrm{M}_{B} \rightarrow \mathrm{M}_{A}$ is strong monoidal, i.e., the $\mathcal{H}(P)_{M, N}$ for all objects $M, N$ and the $\mathcal{H}(P)_{0}$ are isomorphisms. Such situations motivate the

Definition 4.3. A comonoid $\langle g, \gamma, \pi\rangle$ in a closed monoidal category $\langle\mathcal{C}, \bigotimes, e, \mathbf{a}, \mathbf{l}, \mathbf{r}\rangle$ is called a strong comonoid if the monoidal functor

$$
\begin{aligned}
\mathcal{H}=\operatorname{Hom}\left(g,{ }_{-}\right): \mathcal{C} & \rightarrow{ }_{T} \mathcal{C}_{T} \\
\mathcal{H}_{a, b}: \operatorname{Hom}(g, a) \underset{T}{\otimes} \operatorname{Hom}(g, b) & \rightarrow \operatorname{Hom}(g, a \otimes b), \quad \alpha \underset{T}{\otimes} \beta \mapsto(\alpha \otimes \beta) \circ \gamma \\
\mathcal{H}_{0}: T & \rightarrow \operatorname{Hom}(g, e), \quad \tau \mapsto \tau
\end{aligned}
$$

is strong. Here $T$ is the convolution monoid $\operatorname{Hom}(g, e)$ and for each object $a$ the $\operatorname{Hom}(g, a)$ is given the $T$-T-bimodule structure $\tau \cdot \alpha=\mathbf{l}_{a} \circ(\tau \otimes \alpha) \circ \gamma, \alpha \cdot \tau=$ $\mathbf{r}_{a} \circ(\alpha \otimes \tau) \circ \gamma$.

For $P$ a strong comonoid in $\mathrm{M}_{B}$ there is a bialgebroid $\left\langle E, T, s_{E}, t_{E}, \Delta_{E}, \varepsilon_{E}\right\rangle$, called the endomorphism bialgebroid, defined as follows.

$$
\begin{aligned}
E & :=\operatorname{End}_{B}(P) \quad \text { as an algebra } \\
T & :=\operatorname{Hom}_{B}(P, S) \quad \text { with multiplication } \tau \star \tau^{\prime}:=\left(\tau \underset{S}{\otimes} \tau^{\prime}\right) \circ \Delta_{P} \\
s_{E} & : \tau \mapsto(P \underset{S}{\otimes} \tau) \circ \Delta_{P} \\
t_{E} & : \tau \mapsto\left(\tau \otimes_{S} P\right) \circ \Delta_{P} \\
\Delta_{E} & : \alpha \mapsto \mathcal{H}_{P, P}^{-1}\left(\Delta_{P} \circ \alpha\right) \\
\varepsilon_{E} & : \alpha \mapsto \varepsilon_{P} \circ \alpha,
\end{aligned}
$$

If $P_{B}$ is the restriction of a $P: B \rightarrow A$ then the $T$ and $R$ actions on $E$ are related by the isomorphism $\mathcal{H}(P)_{0}: R \stackrel{\sim}{\longrightarrow} T$. Namely, for $r \in R, \tau=\varepsilon_{P}(r$. $)$ we have

$$
\begin{aligned}
& \tau^{\cdot} \alpha=\alpha \circ t_{E}(\tau)=\alpha(\ldots r) \equiv r \cdot \alpha \\
& \alpha \cdot \tau=\alpha \circ s_{E}(\tau)=\alpha\left(r .{ }^{\prime}\right) \equiv \alpha \cdot r .
\end{aligned}
$$

Lemma 4.4. If the bialgebroid morphism $P: B \rightarrow A$ is such that $\mathcal{H}(P)=$ $\operatorname{Hom}_{B}\left(P,_{-}\right)$is a strong monoidal functor then

(1) $E=\operatorname{End}_{B}(P)$ is a bialgebroid with the structure maps given above,

(2) with the natural action $\alpha \triangleright p=\alpha(p)$ of $E$ on $P$ the triple $\left\langle_{E} P_{B}, \Delta_{P}, \varepsilon_{P}\right\rangle$ is a bialgebroid morphism $B \rightarrow E$ and

(3) the map $\lambda: A \rightarrow E$ given by ${ }_{A} P$ is a map of bialgebroids. 
Proof. (1) This follows by patiently substituting (4.1)-(4.6) into the (right) bialgebroid axioms of [15.

(2) $P$ is an $E$ - $B$-bimodule by construction of $E$. The coring structure of $P$ is compatible with the $B$-action since $P$ is a bialgebroid morphism from $B$. So we are left with proving compatibility with the $E$-action, i.e., equations (3), (4), (5) of Lemma 2.2

$$
\begin{aligned}
& \tau \cdot p^{(1)} \otimes_{S} p^{(2)}=p^{(1)} \cdot \tau\left(p^{(2)}\right) \underset{S}{\otimes} p^{(3)}=p^{(1)}{\underset{S}{\otimes}} \tau\left(p^{(2)}\right) \cdot p^{(3)} \\
& =p^{(1)} \underset{S}{\otimes} p^{(2)} \cdot \tau \\
& \alpha^{(1)}\left(p^{(1)}\right) \underset{S}{\otimes} \alpha^{(2)}\left(p^{(2)}\right)=\left(\alpha^{(1)}{\underset{S}{S}}^{(2)}\right) \circ \Delta_{P}(p) \\
& =\Delta_{P}(\alpha(p)) \\
& \varepsilon_{P}(\alpha(p))=\varepsilon_{E}(\alpha)(p)=\varepsilon_{E}(\alpha)\left(p^{(1)}\right) \varepsilon_{P}\left(p^{(2)}\right) \\
& =\varepsilon_{P}\left(\varepsilon_{E}(\alpha)\left(p^{(1)}\right) \cdot p^{(2)}\right)=\varepsilon_{P}\left(\varepsilon_{E}(\alpha) \cdot p\right) .
\end{aligned}
$$

(3) The map $\lambda: a \mapsto a \triangleright$ _ is an algebra map obviously. Let $\lambda_{0}:=\varepsilon_{E} \circ \lambda \circ s_{A}$ : $R \rightarrow T$ then $\lambda_{0}(r)=\varepsilon_{P}\left(r ._{-}\right)=\mathcal{H}(P)_{0}(r)$, thus $\lambda_{0}=\mathcal{H}(P)_{0}$ is an isomorphism. Now it is clear that

$$
\begin{aligned}
& \lambda \circ s_{A}(r)=r .{ }_{-}=s_{E} \circ \lambda_{0}(r) \\
& \lambda \circ t_{A}(r)={ }_{-} \cdot r=t_{E} \circ \lambda_{0}(r) .
\end{aligned}
$$

Using compatibility of $\Delta_{P}$ with the $A$-action, then the restricted naturality of $\mathcal{H}(P)_{P, P}$ we obtain

$$
\begin{aligned}
\Delta_{E}(\lambda(a)) & =m^{-1}\left(\Delta_{P} \circ \lambda(a)\right)=m^{-1}\left(\left(\lambda\left(a^{(1)}\right) \underset{S}{\otimes} \lambda\left(a^{(2)}\right)\right) \circ \Delta_{P}\right) \\
& =\lambda\left(a^{(1)}\right) \underset{T}{\otimes} \lambda\left(a^{(2)}\right)
\end{aligned}
$$

and finally

$$
\varepsilon_{E}(\lambda(a))=\varepsilon_{P} \circ \lambda(a)=\varepsilon_{P}\left(\varepsilon_{A}(a) . .\right)=\lambda_{0}\left(\varepsilon_{A}(a)\right) .
$$

\section{Monoidal Morita equivalence}

In this section we study the properties of bialgebroid morphisms $P: B \rightarrow A$ that give rise to monoidal category equivalences $\mathcal{H}(P): \mathrm{M}_{B} \simeq \mathrm{M}_{A}$. By Lemma 3.3 all monoidal equivalences are obtained in this way.

Forgetting the monoidal structure, at first, we are in the situation of classical Morita theory. The functor $\operatorname{Hom}_{B}\left(P,_{-}\right): \mathrm{M}_{B} \rightarrow \mathrm{M}_{A}$ is an equivalence of categories, so is its left adjoint $-{ }_{A} P: \mathrm{M}_{A} \rightarrow \mathrm{M}_{B}$, therefore ${ }_{A} P_{B}$ is a Morita equivalence bimodule with inverse equivalence bimodule ${ }_{B} Q_{A}=\operatorname{Hom}_{B}(P, B)$. It follows that ${ }_{A} P_{B}$ and ${ }_{B} Q_{A}$ are faithfully balanced bimodules and the $P_{B},{ }_{A} P, Q_{A},{ }_{B} Q$ are all progenerators (i.e., finitely generated projective generators). In particular $A$ is determined up to isomorphism by $P_{B}$ as $\operatorname{End}_{B}(P)$.

The monoidal structure on the functor $\mathcal{H}(P)$ imposes on the progenerator $P_{B}$ a comonoid structure $\left\langle P, \Delta_{P}, \varepsilon_{P}\right\rangle$. Since a monoidal equivalence functor is nothing else but an equivalence functor with a strong monoidal structure, this comonoid must be strong and every strong comonoid progenerator $\left\langle P, \Delta_{P}, \varepsilon_{P}\right\rangle$ determines 
a monoidal equivalence $\operatorname{Hom}_{B}\left(P,_{-}\right): \mathrm{M}_{B} \rightarrow \mathrm{M}_{E}$ where $E$ is the endomorphism bialgebroid of $P$.

Definition 5.1. Over the base category $\mathrm{M}_{k}$ of $k$-modules consider two bialgebroids: $A$ over $R$ and $B$ over $S$. We say that $A$ and $B$ are monoidally Morita equivalent and write $A \simeq B$ if there is a $k$-linear monoidal category equivalence $\mathrm{M}_{A} \simeq \mathrm{M}_{B}$.

Monoidal Morita equivalence is the same as equivalence of objects in the bicategory Bgd.

Lemma 5.2. The Morita equivalence class of a bialgebroid $B$ can be represented in the monoidal module category $\mathrm{M}_{B}$ as follows.

(1) Let $A \simeq B$ and let $P: B \rightarrow A$ be an equivalence in Bgd. Then $\left\langle P, \Delta_{P}, \varepsilon_{P}\right\rangle$ is a strong comonoid progenerator in $\mathrm{M}_{B}$ and $\lambda_{P}: A \rightarrow \operatorname{End}\left(P_{B}\right)$ is an isomorphism of bialgebroids.

(2) If $\left\langle P, \Delta_{P}, \varepsilon_{P}\right\rangle$ is a strong comonoid progenerator in $\mathrm{M}_{B}$ then $\operatorname{End}\left(P_{B}\right) \simeq B$.

Proof. (1) $P$ is an equivalence in $\operatorname{Bgd}$ iff $\mathcal{H}(P)$ is a monoidal equivalence in MonCat. In particular $\mathcal{H}(P)$ is strong monoidal. Thus Lemma 4.4 implies that $P$ is a strong comonoid progenerator and $\lambda_{P}$ is an isomorphism. (2) Since $P_{B}$ is a progenerator, ${ }_{E} P_{B}$ is a Morita equivalence bimodule. Therefore $\mathcal{H}(P)$ is an equivalence of categories and is equipped with a strong monoidal structure. Therefore $\mathcal{H}(P): \mathrm{M}_{B} \rightarrow \mathrm{M}_{E}$ is a monoidal equivalence.

The above characterization of the Morita equivalence class of $B$ would not be very useful without the next proposition which allows one to replace the global property of a comonoid being "strong" with a local one.

Proposition 5.3. Let $F:\langle\mathcal{C}, \otimes, e\rangle \rightarrow\langle\mathrm{M}, \otimes, i\rangle$ be a colimit preserving additive monoidal functor between closed monoidal Abelian categories. We assume also that $\mathcal{C}$ is cocomplete and possesses a generator $g$. Then $F$ is strong monoidal iff the two arrows $F_{g, g}: F g \otimes F g \rightarrow F(g \otimes g)$ and $F_{0}: i \rightarrow F e$ are isomorphisms in $\mathrm{M}$.

Proof. Only the "if" part requires proof. Every object $c$ of $\mathcal{C}$ has a presentation

$$
\coprod^{J} g \stackrel{\alpha}{\longrightarrow} \coprod^{I} g \stackrel{\beta}{\longrightarrow} c \rightarrow 0
$$

for some small sets $I$ and $J$. So the proof consists of two steps: At first we show that $F_{a, b}$ are invertible for $a$ and $b$ being arbitrary coproducts of $g$. At second we show invertibility of $F_{a, b}$ for $a$ and $b$ being cokernels of arrows like $\alpha$ above.

(1) Let $u_{i}: g \rightarrow a, i \in I$ be injections of the coproduct $a$ and assume that invertibility of $F_{g, b}$ has already been proven. The arrows

$$
F g \otimes F b \stackrel{F u_{i} \otimes F b}{\longrightarrow} F a \otimes F b \quad i \in I
$$

are injections of a coproduct in $\mathrm{M}$. The universal property of this coproduct implies that $F_{a, b}$ is the unique arrow making the naturality diagram

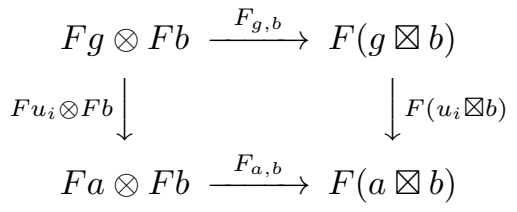


commute. But also the arrows

$$
F(g \otimes b) \stackrel{F\left(u_{i} \otimes b\right)}{\longrightarrow} F(a \otimes b) \quad i \in I
$$

are injections of a coproduct therefore there exists a unique $F_{a, b}^{\prime}$ making

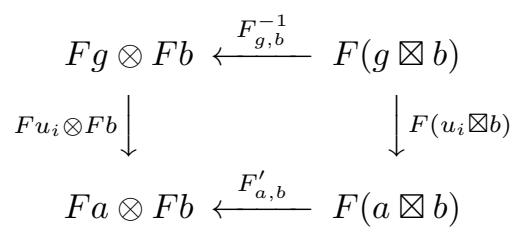

commutative. Now applying the universality properties of the two coproducts again we find that $F_{a, b}^{\prime} \circ F_{a, b}$ and $F_{a, b} \circ F_{a, b}^{\prime}$ are identities, thus $F_{a, b}$ is invertible. Using the above argument at first with $b=g$ we obtain that $F_{a, g}$ is invertible if $a \cong \coprod^{I} g$. Thus, interchanging the roles of the two tensorands, also $F_{g, b}$ is invertible if $b \cong$ $\coprod^{J} g$. Then using the argument at the second time invertibility of $F_{a, b}$ follows for all $a$ and $b$ that are coproducts of $g$.

(2) Let $a \stackrel{\alpha}{\longrightarrow} b \stackrel{\beta}{\longrightarrow} c \rightarrow 0$ be exact where $a \cong \coprod^{I} g, b \cong \coprod^{J} g$ and assume that $d$ is such that invertibility of $F_{a, d}, F_{b, d}$ is already proven. Then the columns of the diagram

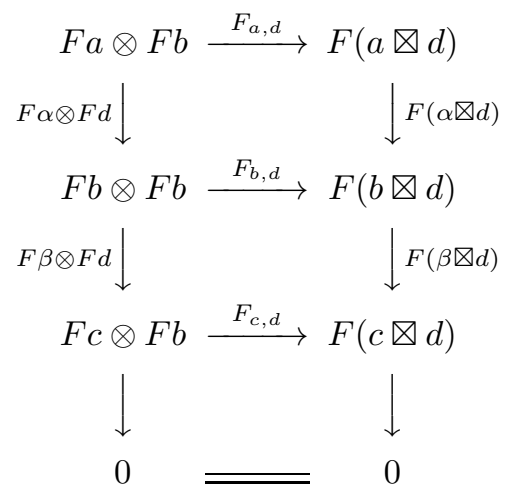

are exact, too. Therefore $F_{c, d}$ is the unique arrow factorizing $F(\beta \bigotimes d) \circ F_{b, d}$ through the cokernel $F \beta \otimes F d$. Similarly, there is a unique arrow factorizing $(F \beta \otimes F d) \circ F_{b, d}^{-1}$ through the cokernel $F(\beta \bigotimes d)$, which is then necessarily the inverse of $F_{c, d}$. Applying this argument at first for $d \cong \coprod^{K} g$ we obtain invertibility of $F_{c, d}$ and, interchanging the roles of the tensorands, of $F_{d, c}$, too. Finally, we can apply the argument for arbitrary object $d$ which finishes the proof.

Corollary 5.4. Let $B$ be a bialgebroid over $S$ and let $\left\langle P, \Delta_{P}, \varepsilon_{P}\right\rangle$ be a comonoid in $\mathrm{M}_{B}$.

(1) If $P_{B}$ is finitely generated projective then $\left\langle P, \Delta_{P}, \varepsilon_{P}\right\rangle$ is a strong comonoid iff

$$
\begin{aligned}
Q \underset{T}{\otimes} Q & \rightarrow \operatorname{Hom}_{B}(P, B \underset{S}{\otimes} B) \\
q \underset{T}{\otimes} q^{\prime} & \mapsto\left(q \underset{S}{\otimes} q^{\prime}\right) \circ \Delta_{P}
\end{aligned}
$$


is an isomorphism, where $Q=\operatorname{Hom}_{B}(P, B), T=\operatorname{Hom}_{B}(P, S)$ and the bimodule structure of $Q$ is given by $(t \cdot q)(p)=t\left(p^{(1)}\right) \cdot q\left(p^{(2)}\right),(q \cdot t)(p)=$ $q\left(p^{(1)}\right) \cdot t\left(p^{(2)}\right)$.

(2) If $P_{B}$ is a progenerator then $\left\langle P, \Delta_{P}, \varepsilon_{P}\right\rangle$ is a strong comonoid iff

$$
\begin{aligned}
E \underset{T}{\otimes} E & \rightarrow \operatorname{Hom}_{B}\left(P, P \otimes_{S} P\right) \\
\alpha \underset{T}{\otimes} \beta & \mapsto(\alpha \underset{S}{\otimes} \beta) \circ \Delta_{P}
\end{aligned}
$$

is an isomorphism, where $E=\operatorname{End}_{B}(P)$ is a $T$-T-bimodule over the convolution monoid $T=\operatorname{Hom}_{B}(P, S)$ as in Subsection 4.3 .

Now we have all the necessary tools to give a characterization of the categories of modules of a bialgebroid.

Theorem 5.5. For a monoidal category $\mathcal{C}$ the following conditions are equivalent:

(1) There is a bialgebroid $A$ and a monoidal equivalence $\mathcal{C} \simeq \mathrm{M}_{A}$.

(2) There is an algebra $R$ and a strong monoidal monadic left adjoint functor $U: \mathcal{C} \rightarrow{ }_{R} \mathrm{M}_{R}$.

(3) $\mathcal{C}$ is cocomplete, Abelian with a small projective generator admitting a strong comonoid structure.

(4) $\mathcal{C}$ is closed, cocomplete, Abelian with a small projective generator admitting a comonoid structure $\langle g, \gamma, \pi\rangle$ such that

$$
\begin{aligned}
& \text { End } g \underset{T}{\otimes} \operatorname{End} g \rightarrow \operatorname{Hom}(g, g \otimes g) \\
& \alpha \underset{T}{\otimes} \beta \mapsto(\alpha \otimes \beta) \circ \gamma
\end{aligned}
$$

is an isomorphism in ${ }_{T} \mathrm{M}_{T}$ where $T=\operatorname{Hom}(g, e)$ with the convolution monoid structure.

Proof. (1) $\Leftrightarrow$ (2) For a bialgebroid $A$ over $R$ the forgetful functor $U: \mathrm{M}_{A} \rightarrow{ }_{R} \mathrm{M}_{R}$ is such a functor. If $R$ and $U$ is given then the bialgebroid $A$ can be reconstructed as the representing object of the opmonoidal monad $\otimes_{R^{e}} A$ on ${ }_{R} \mathrm{M}_{R}$. For details see [16] for left bialgebroids though.

(1) $\Rightarrow(3)$ The $\mathrm{M}_{A}$ is cocomplete Abelian with $A_{A}$ a small projective generator. The coring structure $\left\langle A_{A}, \Delta_{A}, \varepsilon_{A}\right\rangle$ gives the functor $\operatorname{Hom}_{A}\left(A,,_{-}\right): \mathrm{M}_{A} \rightarrow{ }_{R} \mathrm{M}_{R}$ a strong monoidal structure where $R$ is the convolution monoid $\operatorname{Hom}_{A}(A, R)$.

$(3) \Rightarrow(1)$ By Mitchell's Theorem the functor $\operatorname{Hom}\left(g,_{-}\right): \mathcal{C} \rightarrow \mathrm{M}_{A}$ where $A=$ End $g$ is an equivalence of categories if $g$ is a small projective generator. If $g$ has also a strong comonoid structure then the endomorphism bialgebroid construction of Subsection 4.3 equips $A$ with right bialgebroid structure over $R=\operatorname{Hom}(g, e)$ such that $\operatorname{Hom}\left(g,_{-}\right)$is strong monoidal. Hence it is a monoidal equivalence $\mathcal{C} \simeq \mathrm{M}_{A}$.

(4) $\Rightarrow(3)$ Apply Proposition 5.3 to the normal monoidal functor $F=\operatorname{Hom}\left(g,_{-}\right)$: $\mathcal{C} \rightarrow{ }_{T} \mathrm{M}_{T}$ to conclude that $\langle g, \gamma, \pi\rangle$ is strong.

$(3) \Rightarrow(4)$ Since already $(3) \Rightarrow(1)$ and $\mathrm{M}_{A}$ is closed [13, so is $\mathcal{C}$.

Let us briefly discuss the case of Frobenius Hopf algebroids. An invariant for an object $g$ in a monoidal category $\langle\mathcal{C}, \bigotimes, e\rangle$ is an arrow $\iota: e \rightarrow g$. A comonoid $\langle g, \gamma, \pi\rangle$ is called Frobenius if $g$ is selfdual and admits a Frobenius integral. The latter means an invariant $\iota$ such that $\gamma \circ \iota$ is coevaluation for some evaluation $g \otimes g \rightarrow e$ exhibiting $g$ as its own left (and therefore right) dual. This terminology is justified by the observation: Frobenius integrals exists for a comonoid if and only if there 
is an extension of the comonoid structure to a Frobenius algebra $\langle g, \nu, \iota, \gamma, \pi\rangle$ in $\mathcal{C}$. If moreover the comonoid is strong then the endomorphism bialgebroid $E=$ End $g$ becomes equipped with antipode

$$
\begin{gathered}
S_{E}(\alpha)=\mathbf{r}_{g} \circ(g \otimes \varepsilon) \circ(g \otimes \nu) \circ(g \otimes(\alpha \nabla g)) \circ \mathbf{a}_{g, g, g}^{-1} \circ \\
(\gamma \nabla g) \circ(\iota \nabla g) \circ \mathbf{l}_{g}^{-1}
\end{gathered}
$$

so that $E$ is a Hopf algebroid in the sense of [1, 3]. Furthermore, the element $\iota \circ \pi \in E$ is a Frobenius integral in the ordinary sense.This explains how Theorem 5.5 implies the following

Corollary 5.6. For a monoidal category $\mathcal{C}$ the following conditions are equivalent:

(1) There is a Frobenius Hopf algebroid $A$ and a monoidal equivalence $\mathcal{C} \simeq \mathbf{M}_{A}$.

(2) $\mathcal{C}$ is cocomplete, Abelian with a small projective generator admitting a strong Frobenius structure.

(3) $\mathcal{C}$ is closed, cocomplete, Abelian with a small projective selfdual generator $g$ admitting a comonoid structure and a Frobenius integral and such that the map in Theorem 5.5 (4) is an isomorphism.

\section{EXAMPLES}

6.1. Azumaya algebras. Consider the trivial bialgebroid $E(R)$ over $R$ and the trivial bialgebroid over the trivial algebra $E(k) \cong k$. There is a monoidal Morita equivalence $E(R) \simeq E(k)$ iff ${ }_{R} \mathrm{M}_{R} \simeq \mathrm{M}_{k}$ iff $R$ is an Azumaya $k$-algebra by a Theorem of Takeuchi [18]. In this case an equivalence $P: E(R) \stackrel{\sim}{\longrightarrow} E(k)$ can be given as follows. $P$ is the $k$-module $R$ with right $R^{e}$-action $r \triangleleft\left(r_{1} \otimes r_{2}\right)=r_{1} r r_{2}$, with comultiplication $\Delta_{P}(r)=r \otimes 1 \in R \otimes R$ and counit $\varepsilon_{P}(r)=r$. Thus $P$ is nothing else but the monoidal unit of $\mathrm{M}_{E(R)}{ }^{R}$ with its canonical comonoid structure. Therefore it is a strong comonoid. Since an Azumaya algebra is a finite projective and split extension of $k\left[\underline{5}\right.$, the $k$-module ${ }_{k} R$ is a progenerator. By centrality of $R$ the bimodule ${ }_{k} P_{E(R)}$ is faithfully balanced. Therefore $P_{E(R)}$ is also a progenerator. This proves that the monoidal unit $P={ }_{R} R_{R}$ is a strong comonoid progenerator in ${ }_{R} \mathrm{M}_{R}$ for any Azumaya algebra $R$.

6.2. Blowing up. Let $B$ be a bialgebra over $k$ and $n \in \mathbb{N}$. It is well known that $A=$ $\operatorname{Mat}_{n}(B)$ is a weak bialgebra with source and target subalgebras coinciding with $R:=\operatorname{Diag}_{n}(k)$. Now we consider its right bialgebroid version. Right multiplication of $R$ on $A$ makes it an $R$-R-bimodule since $R$ is commutative. Choosing a set of matrix units the coproduct and counit are defined by

$$
\begin{aligned}
\Delta_{A}\left(e_{i j} \otimes b\right) & :=\left(e_{i j} \otimes b^{(1)}\right){\underset{R}{\otimes}}_{\left(e_{i j} \otimes b^{(2)}\right)} \\
\varepsilon_{A}\left(e_{i j} b\right) & :=\delta_{i j} \varepsilon_{B}(b)
\end{aligned}
$$

where $\delta_{i j}$ is the Kronecker symbol.

We would like to show that there is a monoidal Morita equivalence $A \simeq B$. For this purpose let $P=B^{n}$, the column vectors with entries from $B$. It is an $A$ - $B$ bimodule via $(a \triangleright p \triangleleft b)_{i}=\sum_{j} a_{i j} p_{j} b$ and is a Morita equivalence bimodule. Next we make $P$ into a $k$-coalgebra:

$$
\begin{aligned}
\Delta_{P}\left(e_{i} \otimes b\right) & =\left(e_{i} \otimes b^{(1)}\right) \otimes\left(e_{i} \otimes b^{(2)}\right) \\
\varepsilon_{P}\left(e_{i} \otimes b\right) & =\varepsilon_{B}(b)
\end{aligned}
$$


where $\left\{e_{i}\right\}$ is the canonical basis of column vectors. It is easy to verify that (1)-(5) of Lemma 2.2 hold, thus $P: B \rightarrow A$ is a 1-cell in Bgd. It remains to show that the comonoid $P$ is strong. This can be seen directly on the functor $\mathcal{H}(P)$. Indeed, $\operatorname{Hom}_{B}(P, V) \cong \oplus^{n} V$ with the obvious right $A$-module structure. But

$$
\oplus^{n} V \underset{R}{\otimes} \oplus^{n} W \cong \oplus^{n}(V \otimes W)
$$

so $\mathcal{H}(P)$ is strong monoidal. This proves that $P_{B}$ is a strong comonoid progenerator and therefore $A \simeq B$.

6.3. Drinfeld twists. For a bialgebroid $B$ over $S$ a Drinfeld twist can be defined as an invertible element $J \in B \underset{S}{\times} B$ such that

$$
\begin{aligned}
J \eta\left(s \otimes s^{\prime}\right) & =\eta\left(s \otimes s^{\prime}\right) J \quad s, s^{\prime} \in S \\
(J \underset{S}{\otimes} 1)(\Delta \underset{S}{\otimes} B)(J) & =(1 \underset{S}{\otimes} J)(B \underset{S}{\otimes} \Delta)(J) \\
(\varepsilon \underset{S}{\otimes} B)(J) & =1=(B \underset{S}{\otimes} \varepsilon)(J)
\end{aligned}
$$

The 6.1) ensures that the twisted comultiplication $\tilde{\Delta}(b):=J \Delta(b) J^{-1}$ remains an $S$-S-bimodule map. It is easy to show that equations (6.2) and (6.3) imply that $\tilde{\Delta}$ is coassociative and has counit $\tilde{\varepsilon}=\varepsilon$. In this way one obtains the twisted bialgebroid $\tilde{B}$ with the same underlying $S^{e}$-ring structure as $B$.

Now we construct an equivalence $P: B \rightarrow \tilde{B}$. As a $\tilde{B}$ - $B$-bimodule let $P={ }_{B} B_{B}$. The coring structure over $S$ is given by $\Delta_{P}(b):=J \Delta(b)$ and $\varepsilon_{P}(b)=\varepsilon(b)$ for $b \in P$. Coassociativity of $\Delta_{P}$ is clear from (6.2) while counitality can be shown using (6.3) and (1.2). E.g.,

$$
\begin{aligned}
\left(\varepsilon_{P} \underset{S}{\otimes} P\right) \circ \Delta_{P}(b) & =\varepsilon\left(J_{1} b^{(1)}\right) \cdot\left(J_{2} b^{(2)}\right)=J_{2} b^{(2)} t \varepsilon\left(s \varepsilon\left(J_{1}\right) b^{(1)}\right) \\
& =J_{2} t \varepsilon\left(J_{1}\right) b^{(2)} t \varepsilon\left(b^{(1)}\right)=b^{(2)} t \varepsilon\left(b^{(1)}\right)=b .
\end{aligned}
$$

The $\Delta_{P}$ also satisfies (1.2) due to (6.1) and we have

$$
\begin{aligned}
\Delta_{P}\left(b b^{\prime}\right) & =\tilde{\Delta}(b) \Delta_{P}\left(b^{\prime}\right) \\
\varepsilon_{P}\left(b b^{\prime}\right) & =\varepsilon\left(s \varepsilon(b) b^{\prime}\right)=\varepsilon_{P}\left(\tilde{\varepsilon}(b) \cdot b^{\prime}\right)
\end{aligned}
$$

Therefore Lemma 2.2 implies that $P$ is a 1-cell in Bgd from $B$ to $\tilde{B}$. Clearly, $P_{B}$ is a progenerator. In order to see that $P$ is a strong comonoid in $\mathrm{M}_{B}$, let us note at first that $T=\operatorname{Hom}_{B}(P, S) \cong S$ acts on $E=\operatorname{End}_{B}(P) \cong B$ "like" $S$ acts on $B$. At second, we can use the criterion of Corollary $5.4(2)$ as follows. Composing the map there with the tensor square of the isomorphism $\operatorname{End}\left(B_{B}\right) \cong B$ we obtain

$$
\begin{aligned}
B \underset{S}{\otimes} B & \stackrel{\sim}{\longrightarrow} E \underset{T}{\otimes} E \stackrel{\sim}{\longrightarrow} \operatorname{Hom}_{B}(P, P \underset{S}{\otimes} P) \\
b \underset{S}{\otimes} b^{\prime} & \mapsto\left\{b^{\prime \prime} \mapsto b b^{\prime \prime(1)}{\underset{S}{S}}^{\prime} b^{\prime} b^{\prime \prime 2}\right\}
\end{aligned}
$$

which is an isomorphism because $P_{B}$ is cyclic. This proves that $P_{B}$ is a strong comonoid progenerator therefore $E \simeq B$. But also $\lambda_{P}: \tilde{B} \rightarrow E$ is an isomorphism of bialgebroids, hence $\tilde{B} \simeq B$.

Note that any pair of isocategorical groups 4 provides a special case of this example. 
6.4. $\sqrt{\text { Morita }}$ base change. In case of trivial bialgebroids $E(R)$ and $E(S)$ our definition of monoidal Morita equivalence $E(R) \simeq E(S)$ reduces to Takeuchi's $\sqrt{\text { Morita }}$ equivalence of $R$ and $S$ [18. If $B$ is a bialgebroid over $S$ and $P: E(R) \stackrel{\sim}{\longrightarrow} E(S)$ is a monoidal Morita equivalence bimodule then Schauenburg's construction in [14, Theorem 4.3] corresponds to an invertible 2-cell

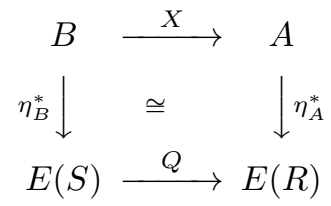

in Bgd where $Q$ is an inverse equivalence of $P$ and the bialgebroid $A$ and the 1-cell $X$ is constructed as follows. In the language of $\operatorname{Cgd}$ the $A$ is the monad

$$
\begin{aligned}
A & :=\left\langle Q \odot B \odot P, \mu_{A}, \eta_{A}\right\rangle \\
\mu_{A} & =A \odot A \stackrel{\sim}{\longrightarrow} Q \odot B \odot B \odot P \stackrel{Q \odot \mu_{B} \odot P}{\longrightarrow} A \\
& \eta_{A}=E(R) \stackrel{\sim}{\longrightarrow} Q \odot P \stackrel{Q \odot \eta_{B} \odot P}{\longrightarrow} A
\end{aligned}
$$

and the $X$ is the bimodule

$$
\begin{aligned}
X & :=\left\langle Q \odot B, \lambda_{X}, \rho_{X}\right\rangle \\
& \lambda_{X}=A \odot X \stackrel{\sim}{\longrightarrow} Q \odot B \odot B \stackrel{Q \odot \mu_{B}}{\longrightarrow} X \\
\rho_{X} & =X \odot B \stackrel{Q \odot \mu_{B}}{\longrightarrow} X
\end{aligned}
$$

The required invertible 2-cell $\eta_{A}^{*} \underset{A}{\otimes} X \rightarrow Q \otimes_{S^{e}} \eta_{B}$ is obtained as follows. As a left $A$-module map $\lambda_{X}$ provides the isomorphism $\eta_{A}^{*} \otimes X \rightarrow X$ of 1-cells in Bgd, on the one hand. On the other hand, $X$ is the horizontal composition $X=Q \otimes_{E(S)} \eta_{B}^{*}$ of 1-cells in Bgd.

The 1-cell $X$ can be easily shown to be an equivalence by constructing an inverse equivalence $Y: A \rightarrow B$. It is left to the reader that $Y=B \odot P$ with its obvious $B$ - $A$-bimodule structure is indeed an inverse equivalence.

We note that bialgebroids $A$ and $B$ can be monoidally Morita equivalent without being there any equivalence $E(R) \simeq E(S)$ between their base bialgebroids, i.e., without their base rings being $\sqrt{\text { Morita }}$ equivalent. A simple example is the blowing up of a bialgebra.

\section{REFERENCES}

[1] G. Böhm, K. Szlachányi, Hopf algebroids with bijective antipodes: axioms, integrals, and duals, J. Algebra 274 (2004) 708-750

[2] T. Brzeziński and G. Militaru, Bialgebroids, $\times_{A}$-bialgebras and duality, J. Algebra 251 (2002) 279-294

[3] B. Day, R. Street, Quantum categories, star autonomy, and quantum groupoids, in "Galois theory, Hopf algebras, and semiabelian categories", Fields Inst. Comm. 43 (2004) 187-225

[4] P. Etingof, S. Gelaki, Isocategorical groups, Internat. Math. Res. Notices 2001, no.2, $59-76$

[5] L. Kadison, New examples of Frobenius extensions, University Lecture Series, Vol. 14, Amer. Math. Soc., Providence, 1999

[6] T. Leinster, Basic bicategories, math.CT/9810017

[7] J.-H. Lu, Hopf algebroids and quantum groupoids, Int. J. Math. 7 (1996) 47-70 
[8] B. Mitchell, Theory of Categories, Academic Press New York - London, 1965

[9] M. Müger, From subfactors to categories and topology. I. Frobenius algebras in and Morita equivalence of tensor categories, J. Pure Appl. Algebra 180 (2003) 81-157

[10] B. Pareigis, Morita equivalence of module categories with tensor product, Comm. Algebra 9 (1981) 1455-1477

[11] B. Pareigis, A non-commutative non-cocommutative Hopf algebra in "nature", J. Algebra 70 (1981) 356-374

[12] P. Schauenburg, Bialgebras over noncommutative rings, and a structure theorem for Hopf bimodules, Applied Categorical Structures 6 (1998) 193-222

[13] P. Schauenburg, Duals and Doubles of Quantum Groupoids, in "New trends in Hopf algebra theory" , Contemporary Mathematics 267 (2000) 273

[14] P. Schauenburg, Morita base change in quntum groupoids, in "Locally Compact Quantum Groups and Groupoids" ed.: L. Vainerman (IRMA Lectures in Mathematics and Theoretical Physics 2) de Gruyter 2003, 79-104

[15] K. Szlachányi, Finite quantum groupoids and inclusions of finite type, Fields Inst. Comm. 30 (2001) 393-407

[16] K. Szlachányi, The monoidal Eilenberg-Moore construction and bialgebroids, J. Pure Appl. Algebra 182 (2003) 287-315

[17] M. Takeuchi, Groups of algebras over $A \otimes \bar{A}$, J. Math. Soc. Japan 29 (1977) 459-492

[18] M. Takeuchi, $\sqrt{\text { Morita }}$ theory - Formal ring laws and monoidal equivalences of categories of bimodules, J. Math. Soc. Japan 39 (1987) 301-336

Research Institute for Particle and Nuclear Physics, Budapest

E-mail address: szlach@rmki.kfki.hu 